\title{
Why Are Self-Report and Behavioral Measures Weakly Correlated?
}

\author{
Junhua Dang ${ }^{1}$ \\ Kevin M. King ${ }^{2}$ \\ Michael Inzlicht ${ }^{3,4}$
}

1 Department of Neuroscience, Uppsala University

2 Department of Psychology, University of Washington

3 Department of Psychology, University of Toronto

4 Rotman School of Management, University of Toronto

Correspondence: dangjunhua@gmail.com (J. Dang)

Keywords: Jingle-Jangle Fallacy; Reliability Paradox; Between-Person Variability

This paper has been accepted by Trends in Cognitive Sciences as a Forum article. This is an early version submitted to TiCS. For the final version after revision according to the comments of the editor and the reviewers, please see the published version.

(https://www.sciencedirect.com/science/article/abs/pii/S1364661320300255) 


\begin{abstract}
Accumulating evidence indicates weak correlations between self-report and behavioral measures of the same construct. We suggest these weak correlations result from poor reliability of many behavioral measures and distinct response processes involved in the two measurement types. We also describe how researchers can benefit from appropriate use of these measures.
\end{abstract}

Self-report and behavioral measures are two of the most popular methods of capturing individual differences in psychology. The same psychological construct is often assessed with both types of measures, with researchers using them interchangeably, often conflating findings across measurement type. However, across a series of domains, recent meta-analyses and large-scale investigations have consistently found self-report and behavioral measures of the same construct were weakly correlated. For example, the average correlation between self-report and behavioral measures of self-control [1], emotional intelligence [2], empathy [3], risk preference [4], and creativity [5] ranged from 0 to 0.20 , indicating a weak association between these two types of measures of the presumed 'same' construct.

This weak association suggests that self-report and behavioral measures might be inherently different and thus cannot be considered interchangeable indicators of a single construct. Our goal here is to 1) explain why self-report and behavioral measures are bound to be weakly correlated by paying careful attention to the psychometric properties of these measures and 2) describe how researchers can benefit from appropriate use of them in research. 


\section{The Reasons for Weak Correlations}

The reasons for weak correlations are both methodological and conceptual.

\section{Reliability Paradox}

Methodologically, the design of many behavioral measures inherently reduces their reliability because they maximize within-person variance at the expense of between-person variance [6]. These behavioral tasks were originally developed to produce robust and replicable experimental effects (via within-person contrasts), but were directly translated from experimental paradigms to between-person correlational studies without sufficient psychometric scrutiny. In order to produce robust experimental effects, between-person variability within a condition needs to be as low as possible, which means most (if not all) people respond to the experimental manipulation. For example, the color-naming Stroop task was designed to maximize the (withinperson) difference between congruent (naming the red color of the word RED) and incongruent (naming the red color of the word GREEN) trials, with the result that nearly everyone shows Stroop interference with little (between-person) variability around this interference effect.

Mathematically, variance between individuals is in the denominator in tests of mean difference between conditions, such as the $t$-test and ANOVA, meaning that lower betweenperson variability within conditions produces larger experimental effects. However, because variance between individuals is also in the numerator in measures of reliability, as shown in Formula 1, lower between-person variability hampers reliability of these behavioral measures. Therefore, the reason for their success in producing robust experimental effect becomes the very 
same reason for their failure to achieve good reliability as measures of individual differences (i.e., the reliability paradox) [6].

$$
\text { Reliability }=\frac{\text { Variance between individuals }}{\text { Variance between individuals }+ \text { Error variance }}
$$

Moreover, trial-by-trial variation in performance can also dramatically attenuate reliability as it increases error variance as indicated in Formula 1, which is especially problematic when the number of trials is limited, as is typical in most behavioral measures [7].

As a result, behavioral measures (typically having low reliability) are likely to be weakly correlated with self-report measures (often with high reliability) because reliability of two measures limits the correlations that can be observed between them, with lower reliability leading to weaker observed correlations, as shown in Formula 2.

$$
\text { Sample correlation }={ }^{\text {True' }} \text { correlation } \sqrt{\text { Reliability }(x) * \text { Reliability }(y)}
$$

\section{Divergent Response Processes}

Some behavioral measures have good reliability yet are still poorly associated with selfreport measures [2,3]; furthermore, correcting for low reliability does not always improve correlations between self-report and behavioral measures $[1,4]$. An alternative explanation, therefore, is that self-report and behavioral measures are distinct because they are designed to measure very different response processes. Specifically, behavioral measures tap responses to uncommon stimuli in a specific and highly structured situation, whereas self-report measures ask participants to reflect on their behaviors across a variety of unstructured real-life situations. Moreover, behavioral measures are based on performance such as reaction time and accuracy, 
whereas self-report measures are based on perceptions of performance, which reflects subjective judgments about performance rather than performance itself.

For example, both the Stroop task (a behavioral measure) and the trait self-control scale (a self-report measure) have been used to measure individual difference in self-control ability. The Stroop task requires individuals to inhibit their prepotent response of reading or to attend more to their goal of color-naming. It is assumed the attentional control processes in the Stroop share similarity with those processes recruited to engage self-control in response to real-life temptations, but these stimuli (color words) are rarely encountered in daily life, nor do they carry the same meaning as real-life temptations. Although items in the trait self-control scale reflect people's actual responses to some extent (e.g., "I am good at resisting temptation"), they are based on people's self-reflection and memory, which are subject to many reporting biases. Thus even if they share the same name (Box 1), these two measures might assess distinct constructs.

\section{Appropriate Applications in Research}

Understanding these methodological and conceptual differences could inform more appropriate applications of these measures in research. Measures with high reliability (i.e. most self-report measures and select behavioral measures such as the working memory span task) can be useful in individual difference research to predict real-life outcomes; and reliable self-report and behavioral measures may explain incremental variance above each other because they actually assess different constructs. Recently, there is also a trend to search various biomarkers (e.g., event related potentials, fMRI activation patterns, heart rate variability, etc.) by relating them to available individual difference measures. We note, with some caution, that attention must be paid to reliability of these potential biomarkers because recent studies have 
demonstrated poor reliability of many biological measures themselves, such as the fMRI-based functional connectivity [8].

Measures with low reliability resulting from low between-person variance are not suitable for individual difference research. Mathematically, as shown in Formula 2, it is expected that these behavioral measures will be weakly correlated with any other measures. Not only are such tasks weakly correlated with self-report measures, they are weakly correlated with other, supposedly related tasks. This insight may shed light on, for example, the considerable inconsistency regarding the unity and diversity of executive functions [9]. For the same reason, these tasks should also have poor predictive validity for real-life outcomes (Box 2).

Despite these notable drawbacks, these measures still have utility. For example, because they are sensitive to within-person experimental manipulations, they can be important for studying the processes that underlie task performance or the contexts that enhance or detract from task performance. They may also be useful for predicting short-term waxing and waning of an attribute for the same individual. For example, an experience sampling study found state performance on a Go/Nogo task predicted snack consumption in the following hour (i.e., withinperson effect), although individual differences in performance on the same task did not predict snack consumption (i.e., between-person effect) [10].

\section{Conclusion}

The weak correlations between self-report and behavioral measures of the presumed same construct result from poor reliability of many behavioral measures and distinct response processes involved in these two measurement types. We suggest that only measures with high 
reliability be used for individual difference research, whereas measure with low reliability may help to predict short-term waxing and waning of an attribute for the same individual. 
Box 1. Low Between-Person Variability and the Jingle-Jangle fallacy

The jingle-jangle fallacy refers to the tempting but often erroneous assumption that two measures with the same name tap the same construct (the jingle fallacy) or two measures with different names tap different constructs (the jangle fallacy). The current article mainly focuses on the jingle fallacy and the contribution of low between-person variability to this fallacy. Low between-person variability, however, may also contribute to the jangle fallacy. For example, the Black-White implicit association test (IAT), a behavioral task, is thought to assess implicit racial bias that is not amenable to consciousness and thus distinct from explicit racial bias. One basis of this claim is the lack of any meaningful association between the Black-White IAT and explicit measures of Black-White attitudes. Recent analyses, however, suggest that the weak correlation between the IAT and explicit racial bias results mainly from the IAT's failure to capture between-person variability [11]. This leaves an uncomfortable question: Despite being thought to reflect the unique construct of implicit bias, does the IAT mostly reflect explicit bias? Regardless of whether the IAT commits the jangle fallacy, it appears clear that it is a poor measure of individual differences in attitudes (implicit or otherwise) due to low between-person variance. 


\section{Box 2. Implications for Executive Functions}

Executive functions are measured by various behavioral tasks with low between-person variance [6]. Mathematically, these measures should thus be weakly correlated with each other and reallife outcomes, which should be observed in studies with sufficient statistical power where the false discovery rate is minimized. However, because many studies are conducted with small samples, observed correlations will tend to be heterogeneous. This has played out in at least two ways: confusion about the structure of executive functions and apparent associations between executive functions and real-world outcomes that disappear with powered samples. Regarding the structure of executive functions, although three factors (i.e., updating, inhibition, and taskswitching) have been identified to represent executive functions by early research, follow-up studies failed to replicate this structure, with the number of identified factors ranging from a single factor to as many as five factors [9]. This inconsistency can be partly explained by low between-person variance that produces heterogeneous results in modeling these tasks in small sample studies. Similarly, regarding predictive validity, early studies using underpowered designs found mixed results for the association between executive functions and real-world outcomes (e.g., dietary intake). Later studies, however, failed to reveal any underlying association once adequate samples were used [12]. 


\section{References}

1 Saunders, B. et al. (2018) Reported self-control is not meaningfully associated with inhibition-related executive function: A Bayesian analysis. Collabra Psychol. 4, 39

2 Joseph, D.L. and Newman, D.A. (2010) Emotional intelligence: An integrative metaanalysis and cascading model. J. Appl. Psychol. 95, 54-78

3 Murphy, B.A. and Lilienfeld, S.O. (2019) Are self-report cognitive empathy ratings valid proxies for cognitive empathy ability? Negligible meta-analytic relations with behavioral task performance. Psychol. Assess. 31, 1062-1072

$4 \quad$ Frey, R. et al. (2017) Risk preference shares the psychometric structure of major psychological traits. Sci. Adv. 3, e1701381

5 Park, N.K. et al. (2016) Revisiting individual creativity assessment: Triangulation in subjective and objective assessment methods. Creat. Res. J. 28, 1-10

6 Hedge, C. et al. (2018) The reliability paradox: Why robust cognitive tasks do not produce reliable individual differences. Behav. Res. Methods 50, 1166-1186

7 Rouder, J.N. and Haaf, J.M. (2019) A psychometrics of individual differences in experimental tasks. Psychon. Bull. Rev. 26, 452-467

8 Noble, S. et al. (2019) A decade of test-retest reliability of functional connectivity: A systematic review and meta-analysis. Neuroimage 203, 116157

9 Karr, J.E. et al. (2018) The unity and diversity of executive functions: A systematic review and re-analysis of latent variable studies. Psychol. Bull. 144, 1147-1185

10 Powell, D.J.H. et al. (2017) Does real time variability in inhibitory control drive snacking 
behavior? An intensive longitudinal study. Heal. Psychol. 36, 356-364

11 Schimmack, U. (2019) The Implicit Association Test: A Method in Search of a Construct. Perspect. Psychol. Sci. DOI: 10.1177/1745691619863798

12 Egbert, A.H. et al. Executive function and dietary intake in youth: A systematic review of the literature. Appetite 139, 197-212 